\title{
Clorose férrica induzida pelo calcário
}

\author{
Maribela Pestana ${ }^{1}$, Amarilis de Varennes ${ }^{2}$, Pedro José Correia ${ }^{1}$ \\ http://dx.doi.org/10.1590/0034-737X201461000010
}

\section{RESUMO}

A clorose férrica é um dos problemas nutricionais mais frequentes e difíceis de controlar nas plantas cultivadas em solos calcários. Nesses solos de reação alcalina, que ocupam cerca de um terço da superfície terreste, prevalece o íon bicarbonato, identificado como sendo um dos principais fatores indutores da clorose férrica. Como resultado, a alcalinidade dificulta a biodisponibilidade do Fe na solução do solo, a redução e a absorção do Fe pela planta, assim como, o respectivo transporte e assimilação desse elemento no metabolismo vegetal. Face a esse desequilíbrio nutricional, as plantas apresentam diferentes estratégias de resposta, que, nessas condições, nem sempre são eficientes. Consequentemente, a produção, a qualidade do fruto e a data de colheita são negativamente afetados. Os gastos com a prevenção e a correção da clorose férrica são elevados e inevitáveis, para assegurar a viabilidade e a sustentabilidade da produção agrícola, nas áreas em que se conjugam a presença de carbonato de cálcio no solo e outros fatores, como os climas áridos ou semiáridos. Com este trabalho, pretende-se resumir a dinâmica do ferro nos solos calcários e suas consequências no metabolismo do Fe e na produção agrícola, com destaque para os frutos.
\end{abstract}

Palavras-chave: bicarbonato, fruteiras, micronutrientes, quelato de ferro redutase

\begin{abstract}
\section{Lime-induced iron chlorosis}

Iron chlorosis is one of the most common and difficult to control problems in crops grown on calcareous soils. In alkaline soils, which represent one third of the Earth surface, the bicarbonate ion prevails and is a major induction factor of iron chlorosis. As a result, alkalinity limits Fe bioavailability in the soil solution, Fe reduction and assimilation, as well as transport and uptake within the plant. Due to this nutritional imbalance, plants develop different response strategies which are not entirely successful on calcareous soils. In consequence, yield, fruit quality and harvesting season are negatively affected. Preventing and treating iron chlorosis is highly costly, but is inevitable, in order to ensure crop sustainability in regions where soil calcium carbonate and aridity are limiting factors. In this work, we present a short overview of Fe dynamics in calcareous soils and its influence on crop productivity.
\end{abstract}

Key words: bicarbonate, fruit-tree, iron-chelate reductase, micronutrients. 


\section{O QUE É A CLOROSE INDUZIDA?}

A clorose induzida pelo calcário identifica-se pelo amarelecimento do limbo foliar e expressa um conjunto complexo de limitações no continuum solo-planta, salientando-se a baixa biodisponibilidade de ferro (Fe) na rizosfera e a restrição na absorção, transporte e, ou, assimilação do Fe pelas plantas (Pestana et al. 2004a). Na folha, o aumento do pH apoplástico conduz à imobilização fisiológica do $\mathrm{Fe}$, que resulta num decréscimo dos pigmentos fotossintéticos e no aparecimento dos sintomas característicos de clorose férrica (Abadía 1992).

Os sintomas foliares iniciam-se pelo aparecimento de um fino reticulado, no qual apenas as nervuras permanecem verdes (Figura 1). Por causa da baixa mobilidade do Fe na planta, esses sintomas surgem primeiro nas folhas mais jovens, originando-se, na mesma planta, um gradiente vertical de cores, relacionado com a concentração foliar de clorofila (Figura 2). Em estados de deficiência mais avançados, os sintomas podem afetar toda a planta, originando a descoloração e necroses das folhas. A evolução dos sintomas de clorose férrica ao longo do tempo pode-se observar em folhas jovens de morangueiros que crescem em solução nutritiva de Hoagland sem Fe (Figura 3). Os sintomas da clorose férrica induzida surgem também em outros órgãos, como se pode constatar nas flores de morangueiro, nas quais se observam as sépalas com clorose férrica avançada (Figura 4).

\section{COMO O CALCÁRIO AFETAA DINÂMICA DO FE NO SOLO?}

Apesar de o Fe ser o quarto elemento mais abundante $(5.1 \%)$ na litosfera, depois do oxigênio, do si-

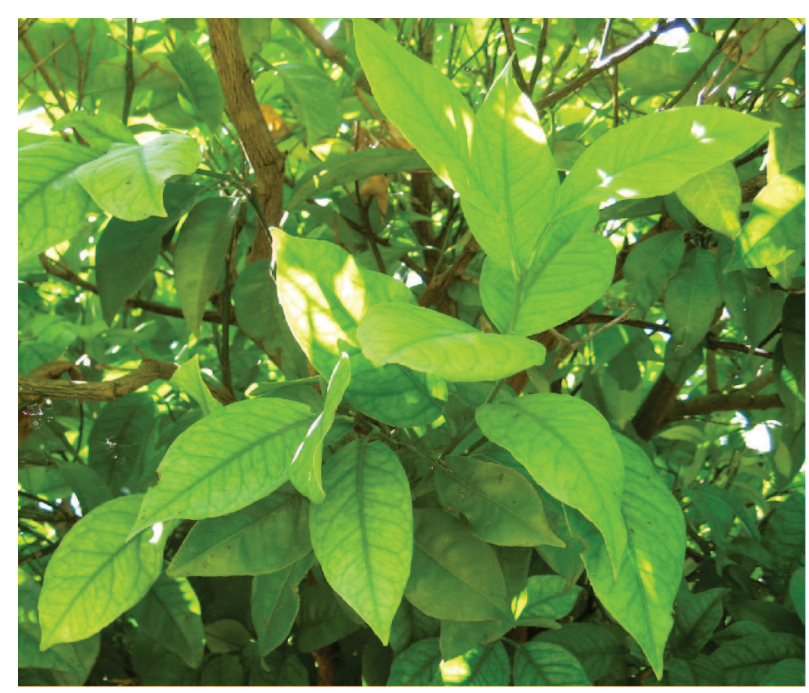

Figura 1. Folhas da laranjeira com sintomas típicos de clorose férrica induzida em solos calcários. lício e do alumínio (Lindsay \& Schwab 1982), a deficiência de Fe é um dos problemas mais antigos e difíceis de controlar, em plantas cultivadas em solos calcários.

A forma de $\mathrm{Fe}$ mais abundante é a hematita $\left(\mathrm{Fe}_{2} \mathrm{O}_{3}\right)$, por sua estabilidade termodinâmica e insolubilidade elevadas (Loeppert 1986). A quantidade total de Fe existente nos vários tipos de solos varia entre $0.02 \%$, nos solos arenosos, até valores superiores a $10 \%$, nos solos ferralíticos (Chen \& Barak 1982). Durante a meteorização das rochas ocorre a precipitação do Fe, em óxidos e hidróxidos, sendo os mais comuns (Sánchez-Alcalá et al. 2012) a goethita $(\alpha-\mathrm{FeOOH})$, as hematitas $(\alpha-$ $\mathrm{Fe}_{2} \mathrm{O}_{3}$ ), a ferrihidrita $\left(\mathrm{Fe}_{5} \mathrm{O}_{8} \mathrm{H} .4 \mathrm{H}_{2} \mathrm{O}\right.$ ), a lepidocrocita (Y$\mathrm{FeOOH})$ e a magnetita $\left(\mathrm{Fe}_{3} \mathrm{O}_{4}\right)$. A solubilidade do $\mathrm{Fe}$ no solo está dependente do potencial redox, de processos de complexação e do pH da solução do solo (Loeppert 1986).

Em condições de cultivo, o potencial de oxidação favorece a formação de $\mathrm{Fe}(\mathrm{III})$, que tem particular tendência a passar a formas insolúveis, não sendo absorvido pela planta. Pelo contrário, em solos sujeitos a condições redutoras, como nos arrozais, prevalece a forma reduzida, que é muito mais solúvel (Schwertmann 1991), e o risco de fitotoxicidade de Fe torna-se superior ao de deficiência. Recentemente, Sánchez-Alcalá et al. (2011) estudaram os efeitos do pré-alagamento de solos calcários, ou anaerobiose induzida, na população microbiana e no aumento da biodisponibilidade do Fe, retardando, assim, o aparecimento dos sintomas de clorose férrica.

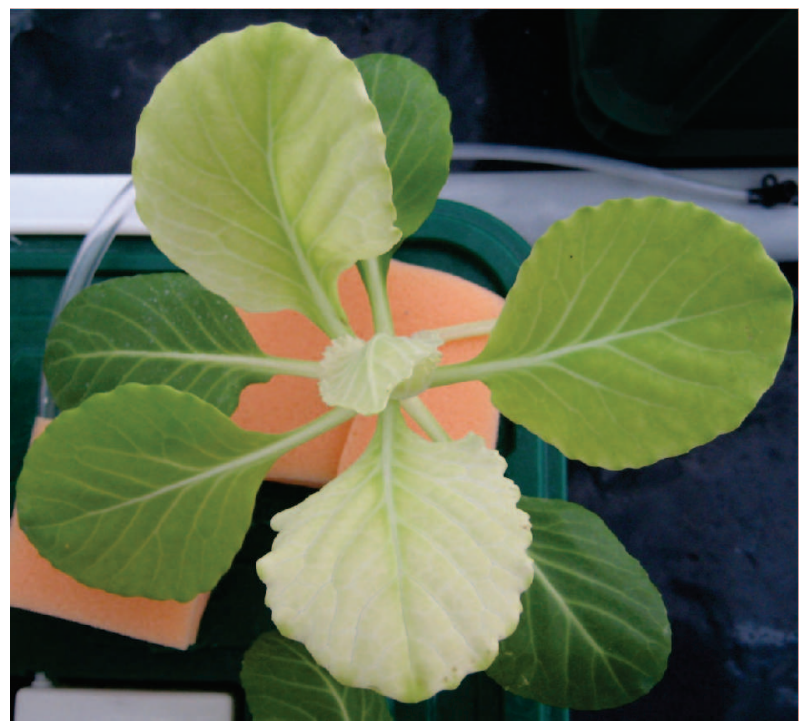

Figura 2. Gradiente vertical dos sintomas de clorose férrica em plantas de couve-galega em hidroponia. Em sentido basípeto: as folhas mais jovens, em desenvolvimento, estão brancas, enquanto as folhas mais velhas (basais) têm a cor verde-escuro característica desta espécie. 
A

Folha verde (sem sintomas)

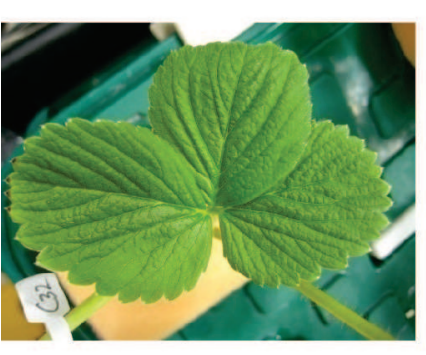

Dia 1
B

Folha com clorose internervuras (sintomas típicos de clorose férrica)

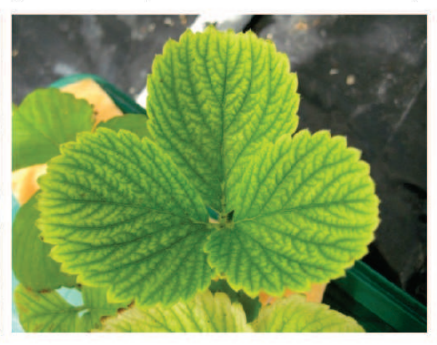

C

Folha com sintomas graves.

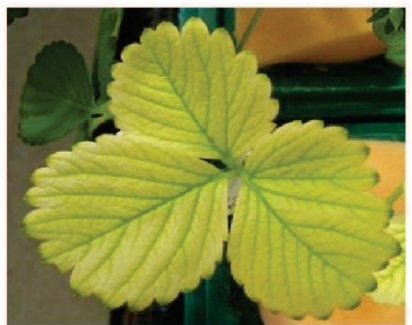

D

Folha branca, que irá cair.

Figura 3. Da esquerda para a direita, folhas novas de morangueiro exibindo agravamento de sintomas de clorose férrica.

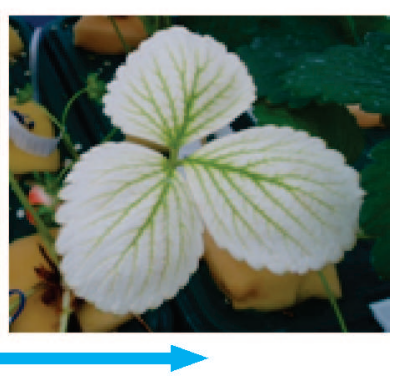

Dia 30
A

Com Fe

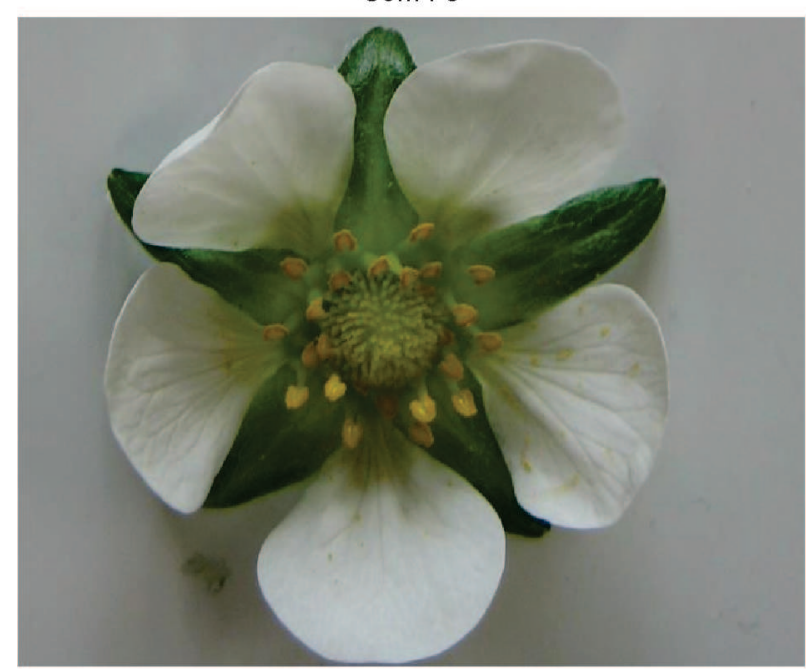

B

Sem Fe

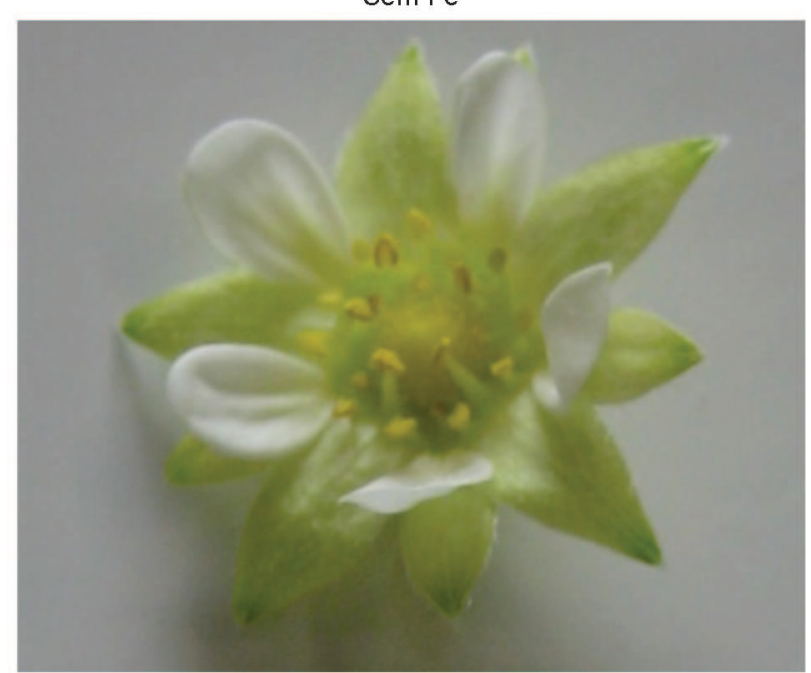

Figura 4. Flores de morangueiro provenientes de plantas verdes (A) e de plantas com clorose férrica (B).

Se, por um lado, os complexantes inorgânicos de $\mathrm{Fe}$ no solo são pouco importantes, por outro os complexos orgânicos podem contribuir para aumentar a mobilidade do Fe no solo (Sánchez-Alcalá et al. 2011). Dentre esses compostos destacam-se os provenientes da degradação da matéria orgânica (ácidos húmicos, ácidos fúlvicos, aminas), os fitosideróforos (exsudados radiculares) e os sideróferos (excretados pelos microorganismos).

A reação do solo determina a presença do aníon bicarbonato $\left(\mathrm{HCO}_{3}^{-}\right)$, soluto predominante nos solos calcários (Eq 1) e principal indutor da clorose férrica. A sua concentração pode frequentemente atingir valores superiores a $200 \mathrm{~g} \mathrm{~kg}^{-1}$ de terra fina e, por apresentar elevado poder tampão, irá anular os prótons (Eq 2) necessários à solubilização dos óxidos férricos (Loeppert 1986):

(Eq 1) $\mathrm{CaCO}_{3}+\mathrm{CO}_{2}+\mathrm{H}_{2} \mathrm{O} \Leftrightarrow \mathrm{Ca}^{2+}+2 \mathrm{HCO}_{3}^{-}$

(Eq 2) $\mathrm{Fe}(\mathrm{OH})_{3}+3 \mathrm{H}^{+} \Leftrightarrow \mathrm{Fe}^{3+}+3 \mathrm{H}_{2} \mathrm{O}$
Os solos calcários localizam-se principalmente nas zonas áridas e semiáridas e ocupam cerca de 1000 milhões de hectares da superfície terreste. Em condições oxidantes, os sais férricos e ferrosos existentes nos solos calcários reagem rapidamente com o $\mathrm{CaCO}_{3}$, originando óxidos de ferro de baixa solubilidade, de acordo com as reações (Eq 3 e 4) propostas por Loeppert (1986).

(Eq 3) $4 \mathrm{Fe}^{2+}+\mathrm{O}_{2}+4 \mathrm{CaCO}_{3}+2 \mathrm{H}_{2} \mathrm{O} \Leftrightarrow 4 \mathrm{FeOOH}+$ $4 \mathrm{Ca}^{2+}+4 \mathrm{CO}_{2}$

(Eq 4) $2 \mathrm{Fe}^{3+}+3 \mathrm{CaCO}_{3}+3 \mathrm{H}_{2} \mathrm{O} \Leftrightarrow=2 \mathrm{Fe}(\mathrm{OH})_{3}+3 \mathrm{Ca}^{2+}$ $+3 \mathrm{CO}_{2}$

Segundo Lindsay \& Schwab (1982), para cada aumento de uma unidade de $\mathrm{pH}$ acima de 7 , a solubilidade do $\mathrm{Fe}$ decresce de 1000 vezes. Para os valores de $\mathrm{pH}$ característicos dos solos calcários (7.5 a 8.5) a concentração de equilíbrio do $\mathrm{Fe}^{3+}$ dissolvido é de aproximadamente $10^{-17} \mathrm{M}$, consideravelmente inferior ao intervalo de valores, entre $10^{-4}$ e $10^{-8} \mathrm{M}$, necessários ao crescimento normal das espécies vegetais (Welch 1995). 


\section{COMO O FE É METABOLIZADO NAS PLANTAS?}

A entrada do Fe na planta requer a sua prévia redução, processo regulado pelas enzimas quelato de $\mathrm{Fe}(\mathrm{III})$ redutases (QF-R), que existem ao longo do percurso do $\mathrm{Fe}$, desde as raízes às folhas, onde o $\mathrm{Fe}$ participa ativamente no metabolismo vegetal.

Quando existe em quantidade suficiente, o Fe entra na planta através de um sistema de redução ligado a um sistema de transporte de baixa afinidade, presente nas membranas plasmáticas (Brüggemann et al. 1990). No entanto, face a uma situação de deficiência, as plantas podem permanecer indiferentes (plantas ineficientes) ou ativar mecanismos fisiológicos de resposta (plantas eficientes), que são designados, no seu conjunto, por Estratégia I, ou estratégia redutora, encontrada nas dicotiledôneas e em monocotiledôneas não gramíneas, e por Estratégia II, ou estratégia complexante, específica das gramíneas (Römheld 1987).

As plantas da Estratégia I, entre as quais se incluem as espécies com interesse agrícola, além de aumentarem a taxa de redução e de absorção radicular do Fe, também acidificam a rizosfera, por meio da excreção de prótons. Apresentam, ainda, alterações morfológicas no sistema radicular. Macroscopicamente, é possível observar o incremento do número de segmentos radiculares mais curtos (Figura 5), aumentando a probabilidade de o Fe entrar na planta. O engrossamento radicular deve-se, em nível microscópico, ao aparecimento de células de transferência, em simultâneo com a acumulação de ácidos orgânicos de baixo peso molecular, como os ácidos cítrico e málico (Rombolà et al. 2002; Abadía et al. 2002; Correia et al. 2014). Os sinais bioquímicos que desencadeiam essas respostas permanecem em discussão, sendo frequentemente referidos diversos hormônios, ou percursores, o óxido nitroso e o próprio elemento (Abadía et al. 2011).

No entanto, esses mecanismos, per si, não são suficientes para assegurar o Fe às culturas agrícolas quando desenvolvidas em solos calcários, pois o efeito tampão do íon bicarbonato interfere com esta estratégia redutora.

Na Figura 6, é possível observar, pela intensidade da cor rosa do complexo Fe-BPDS, a estratégia redutora e os locais de redução do sistema radicular de plantas de alfarrobeira (Ceratonia siliqua L.), lenhosa adaptada a solos calcários (Pestana et al. 2012).

Depois de ultrapassar as barreiras químicas do solo e ser absorvido pela raiz, o Fe atravessa radialmente as células corticais, em direção aos vasos xilematicos. De acordo com Rombolà et al. (2002), o ácido cítrico pode ser o complexante preferencial para o Fe, apenas quando todos os outros ácidos orgânicos existentes no xilema estejam na mesma concentração; quando esta proporcionalidade se altera, é natural que outros ácidos orgânicos consigam também complexar o Fe, como é o caso do ácido málico, que foi detectado em concentrações elevadas na seiva xilêmica de várias espécies vegetais.

O fluxo de Fe translocado para a parte aérea através do xilema é regulado pela transpiração, pelo transporte passivo e, em menor escala, pela pressão radicular, transporte ativo (Welch 1995). A transpiração regula essencialmente o transporte de Fe para as folhas jovens totalmente expandidas, que não apresentam grandes necessidades desse elemento. Por sua vez, o transporte do Fe regulado pela pressão radicular, confinado a períodos de baixa transpiração (noturnos), é direcionado para as zonas de crescimento na parte aérea (ápices caulinares, folhas, frutos e sementes em desenvolvimento), com elevadas necessidades de Fe. O Fe, translocado na forma de citrato férrico, uma vez na parte aérea, é novamente reduzido antes da entrada no mesófilo das células foliares (Nikolic \&Römheld 1999).

O Fe participa em diversos processos metabólicos das plantas superiores (para uma revisão, ver Abadía et al. 2011), nomeadamente, na fotossíntese, na respiração, na fixação do nitrogênio atmosférico e na redução dos íons nitrato, pois pertence ao grupo dos metais de transição, o que lhe confere a capacidade de mudar de valência, modificando o seu estado de oxirredução, e de

\section{B}

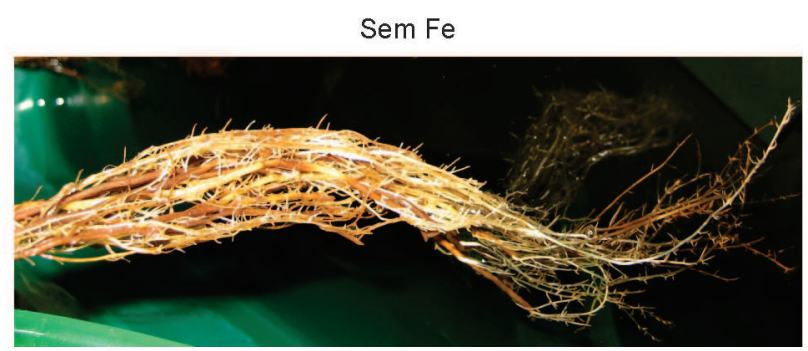

Figura 5. Alterações morfológicas das raízes de morangueiro quando em situação de deficiência de Fe (B) em comparação com raízes desenvolvidas durante o mesmo intervalo de tempo, em solução nutritiva com Fe (A). Destaca-se o elevado número de ápices curtos quando em deficiência de ferro (B).

Rev. Ceres, Viçosa, v. 61, Suplemento, p. 849-855, nov/dez, 2014 
formar complexos octaédricos com várias ligações (Ex.: ácidos orgânicos e fosfato inorgânico).

Em situações normais, a maior parte do Fe foliar (cerca de 63\%) está associada a proteínas, que se agrupam em três categorias (Miller et al. 1995): i) proteínas com grupo heme, nas quais se incluem os citocromos, a catalase, a peroxidase e a nitrato redutase, correspondendo a cerca de $9 \%$ do Fe foliar; ii) proteínas sem grupo heme, como a ferrodoxina, nitrogenase, nitrito redutase, aconitase, xantina oxidase, totalizando cerca de $19 \%$; iii) a fitoferritina, uma proteína de reserva que contém cerca de $35 \%$ do Fe existente nas folhas.

\section{QUAL O EFEITO DA CLOROSE INDUZIDA NA PRODUÇÃO?}

A biodisponibilidade do Fe é o fator limitante para diversas fruteiras estabelecidas em solos calcários, nomeadamente os citrinos, a vinha, o pessegueiro, a pereira, o kiwizeiro, a macieira, entre outras (Tagliavini et al. 2000). Ao afetar diversos processos metabólicos, a clorose férrica é responsável pelos decréscimos observados na produção, no calibre e na qualidade interna do fruto, podendo atrasar a maturação e, consequentemente, a data de colheita. A solução passa pelo fornecimento de Fe a essas culturas, sendo a forma quelatada a mais eficiente (para uma revisão, ver Lucena et al. 2009). As

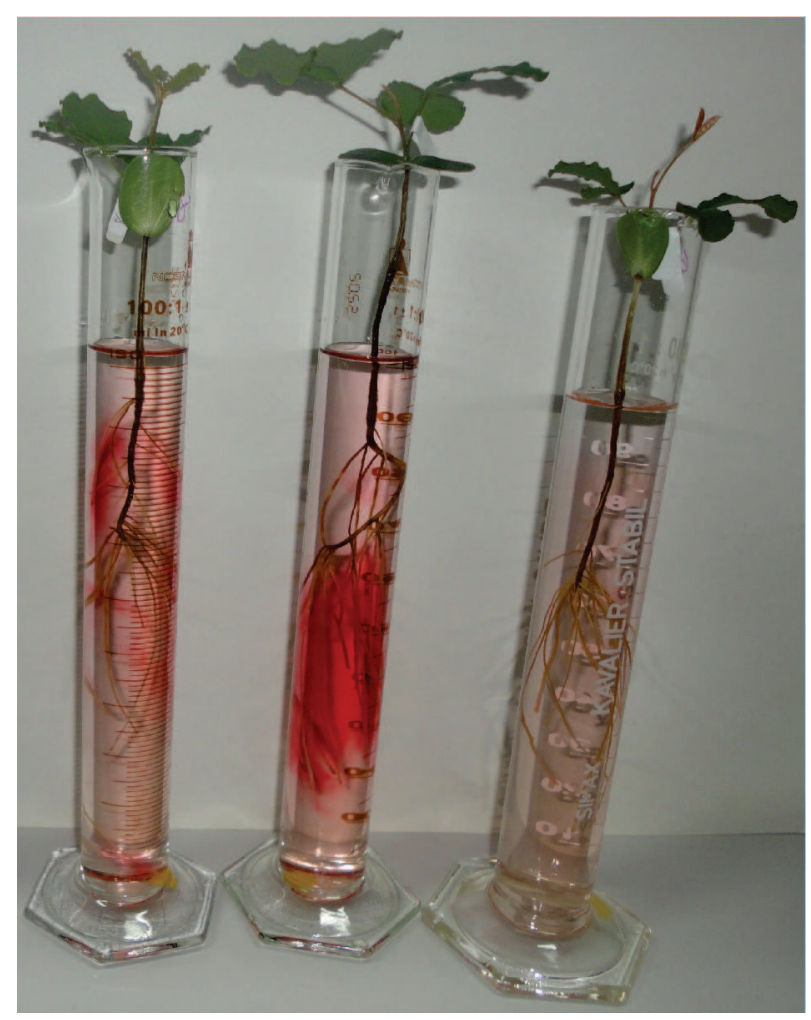

Figura 6. Sistema radicular de alfarrobeiras em provetas de $1000 \mathrm{ml}$ com destaque para a intensidade da cor rosa que é proporcional à quantidade de Fe reduzido e complexado pelo BPDS (bathophenantroline disulfonate). perdas de rendimento associadas a estes sistemas de cultivo são elevadas e difíceis de estimar, pois, nas árvores de fruto esses tratamentos têm de ser repetidos anualmente, caso contrário há agravamento dos sintomas (Pestana et al. 2010a).

Existem diversas referências aos decréscimos na produção e na qualidade da produção, resultando em frutos menores e mais ácidos. Álvarez-Fernández et al. (2003) observaram que pêssegos recolhidos de árvores cloróticas, mesmo antes de apresentarem alterações externas, registravam já a qualidade química interna afetada, como o teor em ácido ascórbico. A presença continuada de clorose férrica resulta em pêssegos e kiwis impróprios para comercialização (Tagliavini et al. 2000). Salienta-se ainda que o decréscimo da qualidade da produção está diretamente relacionado com a intensidade da clorose férrica, expressa pela clorofila foliar.

Em árvores de fruto, a clorose férrica é um processo complexo, que afeta não só o ciclo reprodutivo do ano, como o dos anos seguintes (Pestana et al. 2003; 2004b; 2010a; El-Jendoubi et al. 2013). Juntamente com a deficiência de $\mathrm{Fe}$ é frequente observar outras alterações nutricionais, como são os casos do $\mathrm{P}$, do $\mathrm{Mg}$, do $\mathrm{K}$ e do Zn nas laranjeiras instaladas em solos calcários (Pestana et al. 2005). Entre as fruteiras mais susceptíveis à clorose férrica podem ser referidas a vinha, os citrinos, o pessegueiro, a pereira, o kiwizeiro, a macieira, a oliveira e o damasqueiro. Após um período de clorose prolongado, sem adição de $\mathrm{Fe}$, as reservas esgotam-se e os efeitos surgem na qualidade interna do fruto, na maturação e na produção total, com elevados custos para os produtores (Pestana et al. 2010a).

\section{COMO ANTEVER O APARECIMENTO DA CLOROSE INDUZIDA?}

Num sistema de produção agrícola sustentável, as análises do solo e da água são determinantes do planejamento da fertilização e da correção do solo, antes da instalação de árvores frutíferas. Mais tarde, na manutenção desses pomares, a interpretação dos resultados obtidos pela análise foliar constitui o meio mais correntemente utilizado para avaliar o estado nutricional das árvores de fruto (Moreno et al. 1998). Em solos calcários, o uso de métodos de diagnóstico baseados na análise foliar, quer do Fe total, quer de frações ativas do Fe (Abadía 1992), apresenta limitações. Frequentemente, as folhas deficientes em Fe apresentam muitas vezes concentrações de Fe superiores às existentes nas folhas verdes (Morales et al. 1998). Em muitos casos, não existe qualquer correlação entre a concentração de Fe nos tecidos vegetais e a clorofila total, que expressa o grau de incidência dessa deficiência (Abadía 1992). Este fe- 
nômeno foi denominado por Römheld (2000) como o "paradoxo da clorose férrica".

Verifica-se que, quanto à concentração de Fe no solo, as limitações são semelhantes, já que a análise do Fe total pode, erradamente, dar a ideia de que existe Fe disponível em quantidade suficiente para as plantas. Neste contexto, atrevemo-nos a alargar o conceito de "paradoxo da clorose férrica" ao solo.

$\mathrm{Na}$ tentativa de colmatar este paradoxo global, são testadas diferentes soluções extratantes de Fe e avaliados outros parâmetros, como o teor de argila e o de calcário ativo, no solo (Benítez et al. 2002, Santiago de et al. 2008), enquanto, na análise das plantas, procuramse órgãos de diagnóstico. Estabeleceram-se ainda modelos que complementam essa informação (Pestana et al. 2004b). Por exemplo, o uso da análise floral como método de prognóstico da clorose férrica foi proposto por diversos autores, pois as flores, assim como as folhas, podem ser utilizadas como órgãos que expressam o equilíbrio nutritivo de toda a árvore. Esta relação foi observada em pessegueiro (Igartua et al. 2000; Sanz et al. 1997), macieira (Sanz et al. 1998), pereira (Sanz \& Montañés 1995), laranjeira (Pestana et al. 2005), entre outras. A importância de se determinar um método de diagnóstico é acentuada pelo efeito da clorose férrica na produção e na qualidade do fruto (Pestana et al. 2005; 2010b).

A importância de entender as estratégias fisiológicas das culturas (Pestana et al. 2011; 2012), o recurso a fertilizantes amigos do ambiente (Lucena 2009) e as práticas complementares de gestão nutricional do pomar, ainda são, hoje em dia, importantes linhas de investigação e de experimentação acerca da clorose férrica induzida, em solos calcários, pelo menos enquanto não é possível recorrer a material vegetal tolerante a esse paradoxo.

\section{CONCLUSÕES E PERSPECTIVAS FUTURAS}

Indubitavelmente que, nos últimos anos, houve um grande avanço no estudo da clorose férrica induzida, em solos calcários. No entanto, muitos aspectos permanecem por conhecer, em especial aqueles que têm a ver com as condições de crescimento em campo e com os mecanismos de resposta e o grau de tolerância das diferentes espécies vegetais. Por que duas fruteiras coabitam o mesmo meio e apenas uma das espécies apresenta sintomas? Qual o interruptor que desencadeia e desliga a cadeia de respostas características desse desequilíbrio nutricional, em campo? As implicações da clorose férrica em solos calcários vão muito além da produção, da economia e do ambiente, pois parece haver repercus- sões sociais e na saúde humana. O antecipar da clorose férrica, recorrendo-se à aplicação de fertilizantes com Fe ou a técnicas de produção integrada, será benéfico para a qualidade interna dos frutos? Será mais adequado aproveitar os mecanismos que a planta desencadeia, em resposta à deficiência de $\mathrm{Fe}$, como técnica sustentável de produção? Questões que permanecem sem respostas adequadas e necessitam de ensaios estabelecidos em campo e que mantêm ativo o desafio de estudar a clorose férrica.

\section{AGRADECIMENTOS}

Este trabalho é financiado por Fundos Nacionais, por meio da FCT - Fundação para a Ciência e a Tecnologia, no âmbito do projeto «PTDC/AGR-PRO/3861/2012». Os autores agradecem às Bolsistas Florinda Gama e Teresa Saavedra pela "dedicação à clorose férrica" e por cederem algumas das fotografias deste trabalho.

\section{REFERÊNCIAS}

Abadía J (1992) Leaf response to Fe deficiency: A review. Journal of Plant Nutrition, 15:1699-1713.

Abadía J, López-Millán AF, Rombolà AD \& Abadía A (2002) Organic acids and Fe deficiency: a review. Plant and Soil, 241:75-86.

Abadía JS, Vázquez R, Rellán-Álvarez, El-Jendoubi H, Abadía A, ÁlvarezFernández A \& López-Millán AF (2011) Towards a knowledge-based correction of iron chlorosis. Plant Physiolology \& Biochemistry, 49:471-482.

Álvarez-Fernández A, Grasa R, Abadía A, Sanz M \& Abadía J (2003) Evaluación agronómica de nuevos quelatos de hierro. Phytoma, 145:30-36.

Benítez ML, Pedrajas VM, Campillo MC del \& Torrent J (2002) Iron chlorosis in olive in relation to soil properties. Nutrient Cycling in Agroecosystems, 62:47-52.

Brüggemann W, Moog PR, Nakagawa H, Janiesch P \& Kuiper JC (1990) Plasma membrane-bound NADH: $\mathrm{Fe}^{3+}$ - EDTA reductase and iron deficiency in tomato (Lycopersicum esculentum $\mathrm{L}$.). Is there a turbo reductase? Physiologia. Plantarum, 79:339-346.

Chen Y \& Barak P (1982) Iron nutrition of plants in calcareous soils. Advances in Agronomy, 35:217-240.

Correia PJ, Gama F, Saavedra T, Silva JP da, Miguel MG, Abadía A, Varennes A de \& Pestana M (2014) Changes in the concentration of organic acids in roots and leaves of carob-tree under Fe deficiency. Functional Plant Biology, 41:496-504.

El-Jendoubi H, Abadía J \& Abadía A (2013) Assessment of nutrient removal in bearing peach trees (Prunus persica L. Batsch) based on whole tree analysis. Plant Soil, 369: 421-437.

Igartua E, Grasa R, Sanz M, Abadía A \& Abadía J (2000) Prognosis of iron chlorosis from the mineral composition of flowers in peach. Journal of Horticultural Science \& Biotechnology, 75:111-118.

Lindsay WL \& Schwab AP (1982) The chemistry of iron in soils and its availability to plants. Journal of Plant Nutrition, 5:821-840.

Loeppert RH (1986) Reactions of iron and carbonates in calcareous soils. Journal of Plant Nutrition, 9:195-214.

Lucena J (2009) El empleo de complejantes y quelatos en la fertilización de micronutrientes. Revista Ceres, 56:527-535. 
Miller GW, Huang IJ, Welkie GW \& Pushnik JC (1995) Function of iron in plants with special emphasis on chloroplasts and photosynthetic activity. In: Abadía J (Ed.) Iron nutrition in soils and plants. Dordrecht, Kluwer Academic Publishers. p.19-28.

Morales F, Grasa R, Abadía A \& Abadía J (1998) Iron chlorosis paradox in fruit trees. Journal of Plant Nutrition, 21:815-825.

Moreno DA, Pulgar G, Víllora G \& Romero L (1998) Nutritional diagnosis of fig tree leaves. Journal of Plant Nutrition, 21:2579-2588.

Nikolic M \& Römheld V (1999) Mechanism of Fe uptake by the leaf symplast: Is the $\mathrm{Fe}$ inactivation in leaf a cause of Fe deficiency chlorosis. Plant and Soil, 215:229-237.

Pestana M, Varennes A de \& Faria EA (2003) Diagnosis and correction of iron chlorosis in fruit trees: a review. Journal of Food Agriculture and Environment, 1:46-51.

Pestana M, Varennes A de \& Faria EA (2004a) Lime-induced iron chlorosis in fruit trees. In: R Dris \& SM Jain (Eds.) Production practices and quality assessment of food crops. Volume 2: Plant mineral nutrition and pesticide management. Dordrecht, Kluwer Academic Publishers. p.171-215.

Pestana M, de Varennes A, Goss MJ, Abadía J \& Faria EA (2004b) Floral analysis as a tool to diagnose iron chlorosis in orange trees. Plant and Soil, 259:287-295.

Pestana M, Beja P, Correia PJ, Varennes A de \& Faria EA (2005) Relationships between floral nutrients and fruit quality in orange trees grown in a calcareous soil. Tree Physiology, 24:761-767.

Pestana M, Varennes A de, Miguel MG \& Correia PJ (2010a) Consequences of iron deficiency on fruit quality in citrus and strawberry. In: Nunes C (Ed.) Environmentally Friendly and Safe Technologies for Quality of Fruits and Vegetables. Faro, Universidade do Algarve. p. 92-96.

Pestana M, Gama F, Saavedra T, Correia PJ, Dandlen S \& Miguel MG (2010b) Evaluation of Fe deficiency on strawberry fruit quality. Acta Horticulturae, 868:423-428.

Pestana M, Correia PJ, David M, Abadía A, Abadía J \& Varennes A de (2011) Response of five citrus rootstocks to iron deficiency. Journal of Plant Nutrition \& Soil Science, 174:837-846.

Pestana M, Gama F, Saavedra T, Varennes A de \& Correia PJ (2012) The root ferric-chelate reductase of Ceratonia siliqua (L.) and Poncirus trifoliata (L.) Raf. respond differently to levels of iron. Scientia Horticulturae, 135:65-67.
Rombolà AD, Brüggemann W, López-Millán AF, Tagliavini M, Abadía J, Marangoni B \& Moog PR (2002) Biochemical responses to iron deficiency in kiwifruti (Actinidia deliciosa). Tree Physiology, 22:869875.

Römheld V (1987) Different strategies for iron acquisition in higher plants. Physiologia Plantarum, 70:231-23.

Römheld V (2000) The chlorosis paradox: Fe inactivation as a secondary event in chlorotic leaves of grapevine. Journal of Plant Nutrition, 23:1629-1643.

Sánchez-Alcalá I, del Campillo MC, Torrent J, Straub KL \& Kraemer SM (2011) Iron (III) reduction in anaerobically incubated suspensions of highly calcareous agricultural soils. Soil Science Society American Journal, 75:2136-2146.

Sánchez-Alcalá I, Bellón F, del Campillo MC, Barrón V \& Torrent J (2012) Application of synthethic siderite (FeCO3) to the soil is capable of alleviating iron chlorosis in olive trees. Scientia Horticulturae, 138:17-23.

Santiago A de, Díaz I, del Campillo MC, Torrent J \& Delgado A (2008) Predicting the incidence of iron deficiency chlorosis from hydroxylamine-extractable iron in soil. Soil Science Society American Journal, 72:1493-1499.

Sanz M, Pascual J \& Machín J (1997) Prognosis and correction of iron chlorosis in peach trees: influence on fruit quality. Journal of Plant Nutrition, 20:1567-1572.

Sanz M, Pérez J, Pascual J \& Machín J (1998) Prognosis of iron chlorosis in apple trees by floral analysis. Journal of Plant Nutrition, 21:16971703.

Sanz M \& L Montañés (1995) Floral analysis: A novel approach for the prognosis of iron deficiency in pear (Pyrus communis L.) and peach (Prunus persica L. Batsch.). In: Abadía J (Ed.) Iron nutrition in soils and plants. Dordrecht, Kluwer Academic Publishers. p.371-374.

Schwertmann U (1991) Solubility and dissolution of iron oxides. In: Y Chen \& Y Hadar (Eds.) Iron nutrition and interactions in plants. Dordrecht, Kluwer Academic Publishers. p.03-27.

Tagliavini M, Abadía J, Rombolà AD, Abadía A, Tsipouridis C \& Marangoni B (2000) Agronomic means for the control of iron chlorosis in deciduous fruit trees. Journal of Plant Nutrition, 23:2007-2022.

Welch RM (1995) Micronutrient nutrition of plants. Critical Reviews Plant Science, 14:49-82. 